\title{
Une année de réorientations, records et coopérations
}

\section{Lars Clarfeld}

Secrétaire général de la SSMIG

Le comité de la SSMIG dresse le bilan d'une année riche en événements: outre la participation à la discussion autour de la (mesure de la) qualité (en médecine), la révision du programme de formation continue en MIG, la révision du cursus de formation postgraduée ainsi que des manifestations et congrès passionnants, une nouvelle stratégie a été adoptée. Par ailleurs, la SSMIG a augmenté l'effectif de ses membres en 2019 et s'est exprimée de manière ciblée et répétée lors de procédures de consultation.

L’année 2019 a été turbulente: les débats autour de la (mesure de la) qualité (en médecine), les exigences élevées relatives à la formation continue et postgraduée dans le domaine de la MIG ainsi que les congrès de la SSMIG ont nécessité dévouement et innovation. Aussi le comité a-t-il développé une stratégie destinée à regrouper les engagements et renforcer le "généralisme». Le secrétariat a également connu plusieurs changements de personnel et restructurations.

\section{«La qualité n'est pas un hasard»}

En 2019, la commission de qualité a débuté, sous une nouvelle présidence, l'élaboration d'une nouvelle stratégie globale en matière de qualité et établi de premiers objectifs ainsi que des champs d'action en faveur de la qualité. Le comité de la SSMIG a adopté en janvier 2020 la nouvelle stratégie qualité de la SSMIG pour 2020 à 2024 sous la devise «La qualité n'est pas un hasard».

Sur recommandation de la commission de qualité, le comité de la SSMIG a décidé, en 2019, de s'engager en collaboration avec mfe et le groupe de travail du projet pilote FMH/assureurs. L'objectif de ce groupe de travail est, compte tenu des nouveaux contrats sur le développement de la qualité avec les assureurs prévisiblement nécessaires en 2021, d'élaborer des mesures pertinentes pour le domaine ambulatoire et de négocier celles-ci avec les assureurs.

En 2019, mfe, la SSMIG et Médecins de l'enfance Suisse ont en outre créé, avec la «délégation politique qualité», un nouvel organe avec lequel ils souhaitent à l'avenir collaborer encore plus étroitement et intégrer la création de la commission fédérale de qualité.

En 2019, dans le cadre de la communauté d'intérêts «Forum des cercles de qualité» et sous la direction de la SSMIG, il a été possible pour la première fois de dispenser des cours communs de formation destinés aux modérateurs et modératrices de cercles de qualité ainsi que de poursuivre l'établissement de la rencontre annuelle de formation continue ayant lieu depuis 2018.

\section{Révision du programme de formation continue en MIG}

La révision du programme de formation continue en Médecine Interne Générale (MIG) a constitué un point essentiel de l'an passé. Celle-ci est entrée en vigueur le $1^{\text {er }}$ juin 2019. Outre des détails mineurs, deux éléments ont principalement été modifiés: Premièrement, des précisions ont été apportées à la définition et à la reconnaissance de la formation continue essentielle. Deuxièmement, les conséquences auxquelles doivent s'attendre les médecins n'observant pas l'obligation de formation continue font désormais l'objet d'une réglementation.

\section{Promotion de la relève}

En 2019, le comité de la SSMIG a également considéré la promotion de la relève comme un sujet essentiel. La commission de promotion de la relève créée à cet effet 


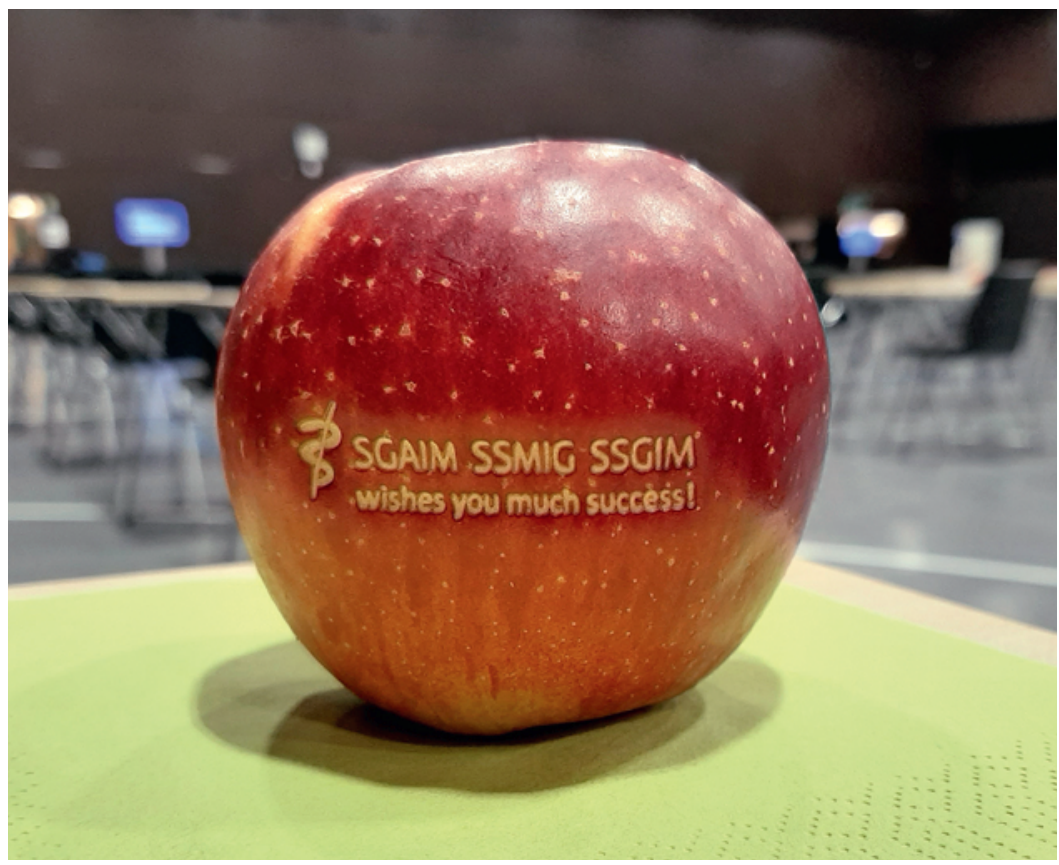

En 2019, I'examen pour l'obtention du titre de spécialiste a de nouveau battu le record de participation. Photo: (c) SSMIG

s'est consacrée en 2019 à l'application d'une nouvelle campagne de promotion de la relève et de publicité. Tandis que la première a notamment pour but de transmettre des images ciblées, de présenter les divers modèles de carrière et de susciter l'intérêt pour la MIG, la campagne publicitaire vise à augmenter la visibilité des spécialistes en Médecine Interne Générale, en particulier des internistes hospitaliers.

En outre, la commission de promotion de la relève a soutenu le développement d'un fil conducteur qui doit faciliter le passage de médecin-assistant à médecin-chef. Martin Perrig et Christine Roten se sont déclarés disposés à prendre en charge ce projet.

\section{Hausse dans le domaine de la formation continue et postgraduée en MIG}

En 2019, l'examen de spécialiste a connu un nouveau record avec au total 892 obtentions du titre de spécialiste en MIG (pour comparaison, chiffres des années passées 2018: 833; 2017: 570; 2016: 678). De même, en 2019, le nombre des diplômes de formation continue dispensés était, avec 2125, plus élevé que jamais (2018: 1431; 2017: 1638).

\section{Nouvelle stratégie décidée}

Lors de la deuxième assemblée des délégués de l'année, la nouvelle stratégie destinée à donner un nouvel essor à la SSMIG a été adoptée. L'accent a été mis sur la créa- tion d'une plus-value pour les membres, l'augmentation de l'attractivité de la MIG en tant que spécialité, la promotion de la qualité et de la recherche ainsi qu'un système tarifaire adapté dans le domaine de la MIG. Une mission, une vision et des valeurs ont par ailleurs été formulées.

\section{Effectif croissant}

L'effectif des membres de la SSMIG a évolué de manière extrêmement satisfaisante. Avec 7516 membres en 2019, la SSMIG a enregistré une hausse d'effectif de 255 personnes. Ce développement positif s'explique également par l'admission de nouveaux membres jeunes.

\section{Participation aux discussions politiques}

En termes de contenu, la SSMIG s'est consacrée à des thèmes très variés lors de diverses procédures de consultation. En 2019, elle a participé à un nombre de procédures de consultation plus élevé que jamais. Cela inclut une prise de position relative au contre-projet indirect à l'initiative sur les soins infirmiers - loi fédérale sur la promotion de la formation dans le domaine des soins infirmiers -, à la consultation sur l'initiative populaire fédérale «Pour sauver des vies en favorisant le don d'organes» et au contre-projet indirect du Conseil fédéral (modification de la loi sur la transplantation).

\section{Congrès attractifs et coopérations passionnantes}

Sous la présidence de l'AMCIS assurée par PD Dr Esther Bächli et Prof. Dr méd. Thomas Fehr, le congrès de printemps a eu lieu du 5 au 9 juin 2019 à Bâle. Il a été pour la première fois renoncé à une version imprimée du programme principal et seule l'application du congrès a été utilisée. Par ailleurs, de nouveaux formats ont été introduits comme par exemple les «mini orals».

Le congrès d'automne 2019 de la SSMIG s'est déroulé du 19 au 20 septembre à Saint-Gall. En collaboration avec KlinFor, les Journées cliniques de la formation continue de l'Hôpital cantonal de Saint-Gall, le congrès a connu un succès retentissant.

A l'occasion du Health Symposium de Berne sous la direction de Prof. Drahomir Aujesky (SSMIG) et Prof. Marcel Zwahlen (PNR 74), des projets actuels du Programme national de recherche 74 ont été présentés et discutés. Le Health Symposium 2019 a attiré près de 80 participants au casino de Berne nouvellement ouvert. 


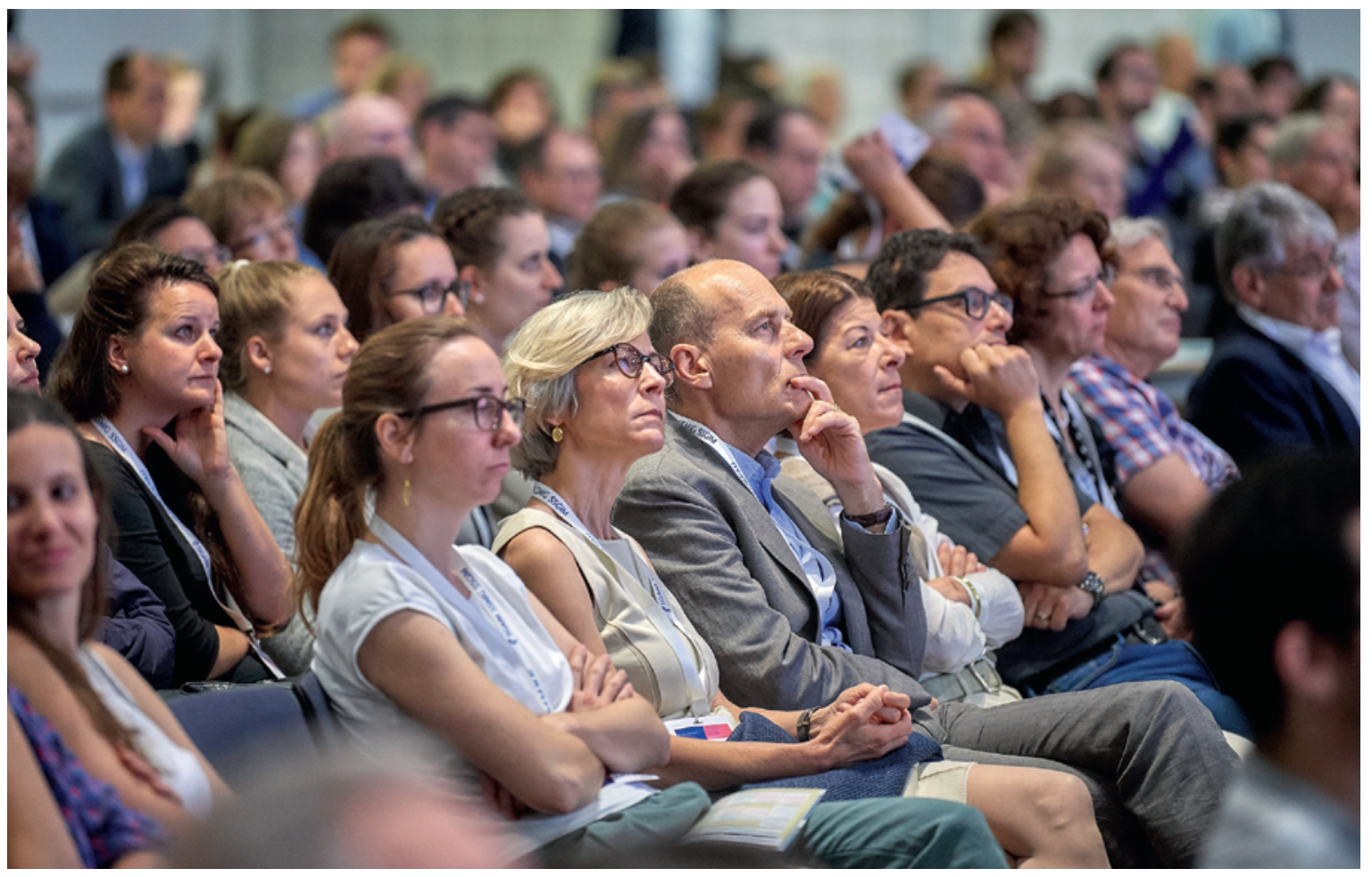

En 2019, de nouvelles offres et coopérations ont contribué à l'attractivité des congrès et manifestations. Photo: (c) SSMIG

\section{Collaborations et synergies}

L'échange avec mfe a été intensifié en 2019. Ainsi, les comités directeurs des deux sociétés se réunissent désormais une fois par mois pour se concerter. La collaboration avec les diverses organisations partenaires Médecins de famille et de l'enfance Suisse (mfe), Association des Médecins-chefs et -cadres Internistes Hospitaliers Suisse (AMCIS), Jeunes médecins de premier recours Suisses (JHaS) et Swiss Young Internists (SYI) a pu être poursuivie dans le cadre actuel.

\section{smarter medicine: «less is more»}

L'association smarter medicine - Choosing Wisely Switzerland, dont le bureau administratif est dirigé par le secrétariat de la SSMIG, a publié en 2019 d'autres listes top 5 des sociétés médicales spécialisées. Des listes supplémentaires sont en cours d'élaboration.
Outre une large couverture médiatique sur le thème du traitement excessif ou erroné en médecine en rapport avec la smarter medicine et les apparitions publiques, une rencontre avec les parlementaires a eu lieu au Café Galerie des Alpes du Palais fédéral.

Par ailleurs, l'association s'est consacrée à une réorientation stratégique: un groupe de travail a déterminé de grands axes et défini notamment les droits et obligations des partenaires. D'autres projets passionnants tels qu'une campagne nationale et des outils d'aide pour le personnel traitant ont été préparés en 2019.
Correspondance:

Responsable communica-

tion et secrétaire

Société Suisse de Médécin

Interne Générale (SSMIG)

Monbijoustrasse 43

Postfach

CH-3001 Bern

claudia.schade[at]sgaim.ch
Le rapport annuel 2019 détaillé du comité est public et peut être téléchargé sur le site Web de la SSMIG sous la rubrique "De la SSMIG / Portrait» (https:// www.sgaim.ch/fr/portrait/portrait.html). 